\title{
New Approach to the Management of Acute Disc Herniation
}

\author{
Arra S. Reddy, MD, Shaun Loh, BS, Jennifer Cutts, MD, Jacob Rachlin, MD, PhD, and Joshua A. Hirsch, MD
}

Background: Over 500,000 percutaneous disc decompression procedures have been performed in the past 20 years. Various percutaneous techniques include chemonucleolysis, percutaneous lumbar discectomy, and laser discectomy which have reported success rates in the $70 \%$ to $75 \%$ range. This retrospective evaluation of 49 patients who underwent nucleoplasty procedures for treatment of herniated discs, evaluates the effectiveness of nucleoplasty in the reduction of pain, improvement of functional activity, and reduction of pain medication.

Objective: To illustrate the effectiveness of nucleoplasty in reducing low back pain in symptomatic patients with contained herniated discs.

The percutaneous decompression of herniated discs is a well-established clinical approach with over 500,000 procedures performed during the past 20 years. Several percutaneous techniques are in practice including chemonucleolysis, percutaneous lumbar discectomy, and laser discectomy. These procedures have re-

From: Departments of Radiology and Neurosurgery, Beth Israel Deaconess Medical Center / Harvard Medical School, and Department of Radiology, Massachusetts General Hospital / Harvard Medical School, Boston, Massachusetts

Address correspondence:

Shaun Loh, Research Fellow

Beth Israel Deaconess Medical Center

Harvard Medical School

One Deaconess Rd., Suite 580

Boston, MA 02215

E-mail: sloh1@bidmc.harvard.edu

Disclaimer: There was no external funding in the preparation of this manuscript.

Conflict of interest: None

Manuscript received on $7 / 10 / 2005$

Revision submitted 8/26/2005

Accepted for publication 9/1/2005

The information in this retrospective review was presented at the Radiological Society of North America 89th Scientific Assembly and Annual Meeting, November 30, 2003 - December 5, 2003 , McCormick Place, Chicago, IL (Educational exhibit o844VI-e)
Study design: A retrospective, non-randomized study.

Methods: Forty-nine patients with either axial or radicular low back pain who had undergone the nucleoplasty procedure were included in this analysis. Patients were categorized in one of three different groups depending on time elapsed since the procedure was performed: less than 6 months, between 6 months and 1 year, and greater than 1 year.

Pain reduction, work impairment, leisure impairment, medication use and patient satisfaction were all recorded during this study. Pain was quantified using a numeric pain scale from o to 10 . Work and leisure impairment were measured on a scale of 1 to 5 , with 1 signifying no impairment and 5 signify- ing extreme impairment. Medication use and patient satisfaction were also measured on a scale of 1 to 5 .

Results: Significant pain relief, functional improvement, and a decrease in medication use were achieved following nucleoplasty. There were no complications associated with the procedure.

Conclusion: Nucleoplasty should be used in those patients who fail conservative medical management including medication, physical therapy, behavioral management, psychotherapy, and who are unwilling to undergo a more invasive technique such as spinal surgery.

Keywords: Nucleoplasty, disc herniation, Coblation, low back pain ported success rates of $70 \%$ to $75 \%$ but each has its limitations. The use of chymopapain showed that disc decompression could be used as a treatment modality for back and leg pain due to herniated discs (1); however, it resulted in an unacceptable level of complications and is no longer available in the United States $(1,2)$. Automated percutaneous lumbar discectomy (APLD) and laser discectomy are in limited use today due to a combination of clinical, design, patient comfort, and cost factors (3-7).

The nucleoplasty procedure is minimally invasive and utilizes Coblation ${ }^{\mathrm{TM}}$ technology to create a plasma field at the tip of the device. The plasma field contains sufficient energy to cleave molecular bonds, thereby ablating tissue. The ablation process creates small channels within the disc, removing that portion of tissue. Coagulation mode is then applied to thermally treat the channels to further decompress the intervertebral disc.

The objective of this study is to illustrate the effectiveness of the nucleoplasty procedure in reducing pain, improving functional activity, and reducing pain medication use in symptomatic patients with contained herniated discs. Several studies have been performed which show that nucleoplasty is effective in reducing pain. One study has shown that nucleoplasty has success rates of up to $80 \%$ with a visual analog scale (VAS) pain score reduction of up to $57 \%$ (8). Other studies showed that $79 \%-85 \%$ of patients reported VAS pain score reductions at 1-, 3, 6-, 9- and 12-month follow-ups $(9,10)$. None of the studies we have reviewed have monitored pain reduction, functional activity, and medication use in nucleoplasty patients more than one year following the procedure.

\section{Methods}

Sixty-seven consecutive patients who had undergone nucleoplasty procedures between January 1, 2002, and May 31,2003 , were selected for this retrospective study. Of the 67 patients contacted, 49 patients completed the survey $(73 \%)$. Of the 18 patients who did not participate, 13 patients could not be reached while the remaining five simply did not want to participate in the study. While there was no inclusion criteria for the study itself, patients were selected to undergo nu- 
cleoplasty based on the presence of either radicular/axial symptoms or axial symptoms. Radicular criteria for inclusion include the following: leg pain > back pain, evidence of contained posterior disc protrusion on MRI, positive discography with concordant pain, or failed selective nerve root block. Axial criteria for inclusion include the following: failed conservative therapy for 3 months, evidence of contained central focal disc protrusion on MRI, or positive discography with concordant pain. Patients were excluded from the study if there was a loss of more than $50 \%$ of disc height, evidence of severe disc degeneration, a fracture or tumor of the spine, or moderate to severe spinal stenosis.

The risks, benefits, and alternatives of the procedure were explained in detail to each patient and consent forms were signed. Each patient was given premedication including $25 \mathrm{mgs}$ of Vistaril, $0.5 \mathrm{mg}$ of Ativan, and $0.5 \mathrm{mg}$ of Xanax approximately 30 minutes before being brought to the neurointerventional suite. In the neurointerventional operating theater, each patient was given a gram of intravenous Ancef ${ }^{\circledast}$. The patient was placed in a prone position on the table, prepped, and draped in the usual sterile fashion.

Using standard techniques, the back was evaluated fluoroscopically and the involved disc space was opened up in the frontal view by rotating in a craniocaudal direction. Under fluoroscopic guidance, a 25 -gauge tuberculin needle followed by a 6-inch, 22-gauge spinal needle, followed ultimately by a 17 -gauge, 6 -inch Crawford needle were used to access the disc. Intravenous contrast was administered when the Crawford needle was placed inside the nucleus pulposus at the junction of the nucleus and the annulus. This discogram was used to confirm discogenic pain or the presence of herniation or degeneration. Through this approach, contrast fills the disc from side-to-side, eventually permeating throughout the entire disc.

Percutaneous discectomy was per-

Table 1. Medication Use Scoring Scale

\begin{tabular}{|r|l|}
\hline 1 & No medications \\
\hline 2 & Occasional non-narcotic \\
\hline 3 & Daily non-narcotic \\
\hline 4 & Occasional narcotic \\
\hline 5 & Daily narcotic \\
\hline
\end{tabular}

formed with the aid of an ArthroCare wand. As suggested by the manufacturer, a total of six passes were performed. Following percutaneous discectomy, the trocar was pulled back into the Crawford needle, and a selective nerve root block was performed using $2 \mathrm{cc}$ of $0.25 \%$ preservative free bupivacaine and $60 \mathrm{mg}$ of methylprednisolone. The needle was removed and a Syvek Patch ${ }^{\oplus}$ placed over the wound site. Fentanyl and Versed were administered during the procedure as part of conscious sedation and were monitored continuously by the radiology nurses.

Several months after the procedure, all of the patients were contacted via phone and were asked questions from a standardized script by a trained volunteer who was not blinded to the study. Patients were asked to quantify pain, how much pain interfered with work and hobbies, pain medication use, and if previous spinal surgeries were performed before the nucleoplasty procedure was performed. Patients were then asked to quantify their pain, work impairment, leisure impairment, and medication use after the nucleoplasty procedure. Finally, patients were asked to quantify their satisfaction level on a scale from 1 to 5 , and asked if they would recommend the procedure to a friend with back pain.

Pain was quantified using a numeric pain scale from 0 to 10 . Work and leisure impairment were measured on a scale of 1 to 5 , with 1 signifying no impairment and 5 signifying extreme impairment. Medication use and patient satisfaction were also measured on a scale of 1 to 5 as shown in Tables 1 and 2 .

The 49 patients were then divided into three separate groups based on the time elapsed since the nucleoplasty was performed: Group 1 - less than six months $(n=16)$; Group $2-$ six months to one year $(\mathrm{n}=22)$; Group 3 - greater than one year $(n=11)$. All procedures were performed under local anesthesia and written informed consent was received from all patients.

Table 2. Patient Satisfaction Scoring Scale

\begin{tabular}{|r|l|}
\hline 1 & Unsatisfactory \\
\hline 2 & Satisfactory \\
\hline 3 & Good \\
\hline 4 & Very Good \\
\hline 5 & Excellent \\
\hline
\end{tabular}

Pre-procedure and post-procedure means, ranges, and standard deviations were calculated. VAS pain score data, work impairment, leisure impairment, and medication use were analyzed using a two tailed paired student t-test with statistical significance set at a $\mathrm{p}$-value $<0.05$.

\section{ResULTS}

\section{Patient Demographics}

Patient demographics are illustrated in Table 3.

\section{Pain Reduction}

On average, there was a 3.67 reduction in VAS score with a mean baseline VAS score of 8.08 .

Figure 1 illustrates the pain score both before the nucleoplasty procedure and after the procedure. A significant reduction in pain in all groups was shown following the nucleoplasty procedure with p-values of $0.000036,0.000009$, and 0.017, for groups 1, 2, and 3 respectively.

\section{Functional Improvement}

Figure 2 illustrates the level of work impairment both before and after the nucleoplasty procedure. A significant reduction in work impairment in all groups was shown following the nucleoplasty procedure with p-values of $0.015,0.00024$, and 0.041 , for groups 1,2 , and 3 respectively.

Figure 3 illustrates the level of leisure impairment both before the nucleoplasty procedure and after the procedure. A significant reduction in leisure impairment in all groups was shown following the nucleoplasty procedure with $\mathrm{p}$-values of $0.019,0.0018$, and 0.029 , for groups 1 , 2 , and 3 respectively.

\section{Pain Medication Use}

Figure 4 illustrates the level of pain medication use both before and after the nucleoplasty procedure. A significant reduction in pain medication use in all groups was shown following the nucleoplasty procedure with p-values of 0.038 , 0.00056 , and 0.035 , for groups 1,2 , and 3 respectively.

\section{Satisfaction}

Most patients surveyed would recommend nucleoplasty to a friend. Group 3 had a 91\% (10/11) recommendation rate, followed by Group 1 at $75 \%$ (12/ $16)$, and Group 2 at 73\% (16/22). All three groups surveyed showed similar satisfac- 
Table 3. Patient demographics

\begin{tabular}{|c|c|c|c|c|c|}
\hline & & Group 1 & Group 2 & Group 3 & Total \\
\hline \multirow{2}{*}{ Gender } & Male & 10 & 16 & 7 & 33 \\
\hline & Female & 6 & 6 & 4 & 16 \\
\hline \multirow{3}{*}{ Age (years) } & Mean & 43 & 45 & 48 & 45 \\
\hline & SD & 12 & 10 & 9 & 11 \\
\hline & Range & $22-67$ & $29-64$ & $34-64$ & $22-67$ \\
\hline \multirow{3}{*}{ Origin of pain } & Trauma & 10 & 5 & 3 & 18 \\
\hline & Work related & 2 & 5 & 2 & 9 \\
\hline & Other & 4 & 12 & 6 & 22 \\
\hline \multirow{3}{*}{ Duration of pain } & Mean & 5 & 5 & 5 & 5 \\
\hline & $\mathrm{SD}$ & 4 & 6 & 6 & 5 \\
\hline & Range & $1-15$ & $1-25$ & $1-20$ & $1-25$ \\
\hline Previous discectomy & & $6 \%(1)$ & $5 \%(1)$ & $2 \%(1)$ & $6 \%(3)$ \\
\hline $\begin{array}{l}\text { Occupation entails } \\
\text { strenuous activity }\end{array}$ & & $31 \%(5)$ & $19 \%(4)$ & $9 \%(1)$ & $20 \%(10)$ \\
\hline \multirow{7}{*}{ Decompression sites } & L2-L3 & $0 \%(0)$ & $5 \%(1)$ & $9 \%(1)$ & $4 \%(2)$ \\
\hline & L3-L4 & $19 \%(3)$ & $9 \%(2)$ & $18 \%(2)$ & $14 \%(7)$ \\
\hline & L4-L5 & $\begin{array}{r}63 \% \\
(10) \\
\end{array}$ & $\begin{array}{r}59 \% \\
(13) \\
\end{array}$ & $18 \%(2)$ & $51 \%(25)$ \\
\hline & L5-L6 & $0 \%(0)$ & $0 \%(0)$ & $9 \%(1)$ & $2 \%(1)$ \\
\hline & L5-S1 & $19 \%(3)$ & $23 \%(5)$ & $45 \%(5)$ & $27 \%(13)$ \\
\hline & T7-T8 & $0 \%(0)$ & $5 \%(1)$ & $0 \%(0)$ & $2 \%(1)$ \\
\hline & Multiple levels & $6 \%(1)$ & $5 \%(1)$ & $18 \%(2)$ & $8 \%(4)$ \\
\hline \multirow{3}{*}{ Classification of pain } & Axillary & $31 \%(5)$ & $32 \%(7)$ & $64 \%(7)$ & $39 \%(19)$ \\
\hline & Radicular & $31 \%(5)$ & $\begin{array}{r}45 \% \\
(10) \\
\end{array}$ & $36 \%(4)$ & $39 \%(19)$ \\
\hline & Both & $38 \%(6)$ & $23 \%(5)$ & $0 \%(0)$ & $22 \%(11)$ \\
\hline
\end{tabular}

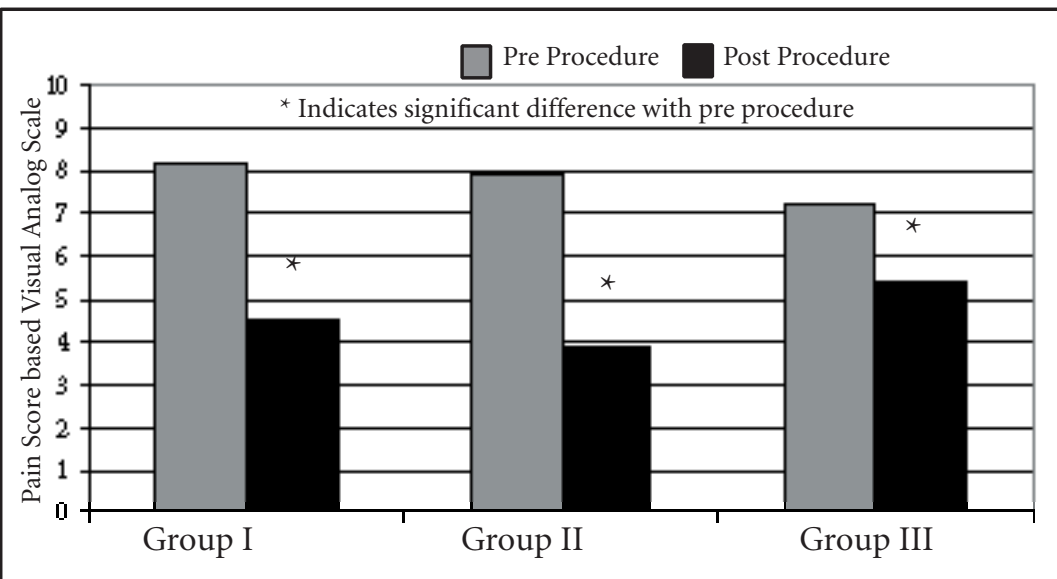

Fig. 1. Pain reduction

tion rates averaging slightly above "good" on the scoring scale $(3.06,3.09$, and 3.27 for groups 1, 2, and 3 respectively).

\section{Complications}

There were no neurological or other complications resulting from the procedure. that pain relief was achieved in $56.4 \%$ ( $9 /$ $16), 64.6 \%(14 / 22)$, and $45.0 \%$ (5/11) for groups 1,2 , and 3 respectively.

Of the seven patients in Group 1 who did not have significant pain relief, four patients went on to have spinal surgery to help with the pain. Only one out of those four patients expressed that they were "unsatisfied" with the nucleoplasty procedure.

In Group 2, eight patients did not report significant pain relief and four patients underwent surgery to alleviate their back pain. All four of the patients who had surgery responded that they were "unsatisfied" with the nucleoplasty procedure.

Of the six patients in Group 3 that did not report significant pain relief, only two went on to receive spinal surgery and both expressed that they were "satisfied" with the nucleoplasty procedure.

The proportion of patients who had achieved at least a $50 \%$ reduction in pain varied among the three groups. It increased from roughly $56 \%$ in Group 1 , to $65 \%$ in Group 2, and decreased greatly to $45 \%$ in Group 3. The general decline in $50 \%$ pain relief over time has been observed in many different interventions for low back pain $(11,12)$. Our VAS pain results are consistent with one other study which showed downward trends in pain scores initially over the first three months, with a steady increase thereafter (8). One thought is that continued trauma, either due to aging or injury, may be continuing in these patients. While the nucleoplasty procedure reduces volume and intradiscal pressure, the underlying cause of the disease may still be present and if not corrected, may continue the progression of the disease.

Our results illustrate that pain reduction is still present more than 1 year after the procedure; however, the decrease in pain relief over time is concerning and further research will have to be performed to isolate the nature of the recurring back pain.

Our study may be criticized because of our study design which included non-randomization of patients, gathering baseline data after the procedure had been performed, and non-blinding of the data during the recording and analysis phase of the study. We will attempt to address each issue.

While we recognize that the gold standard of studies is the randomized, 


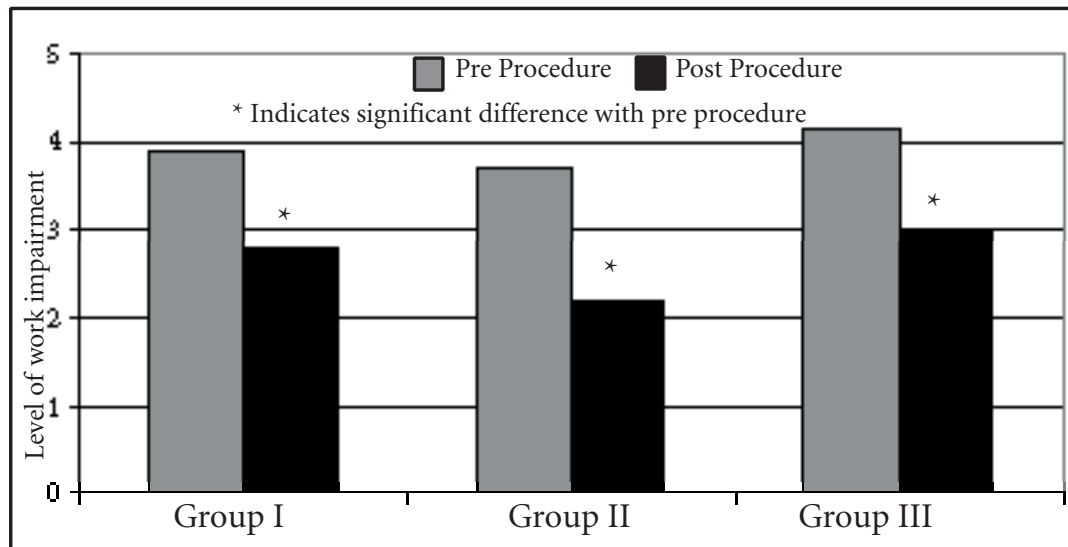

Fig. 2. Work impairment

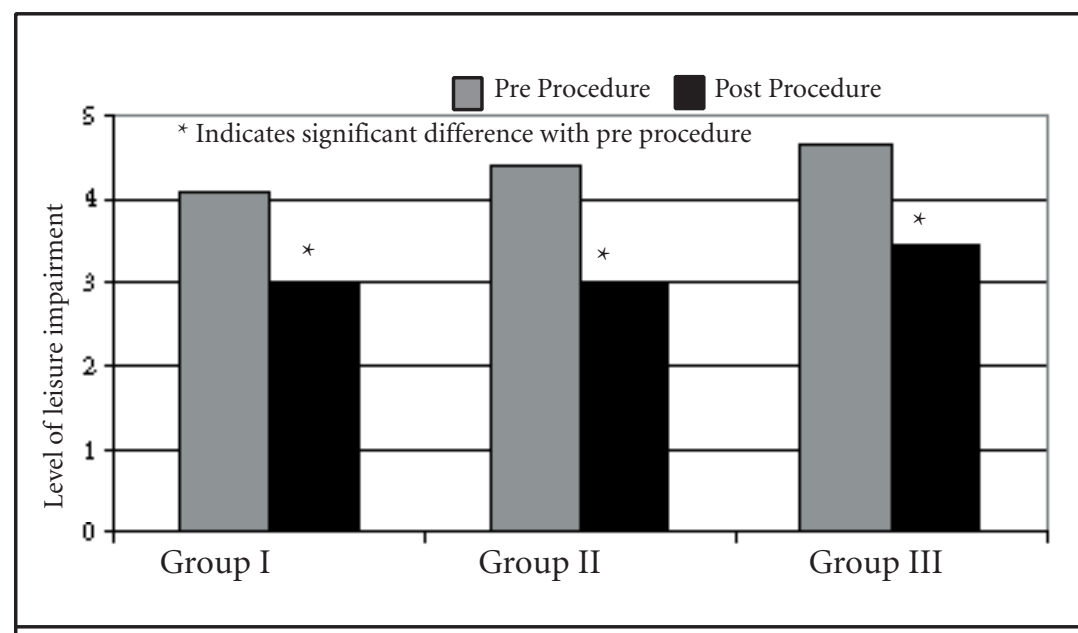

Fig. 3. Leisure impairment

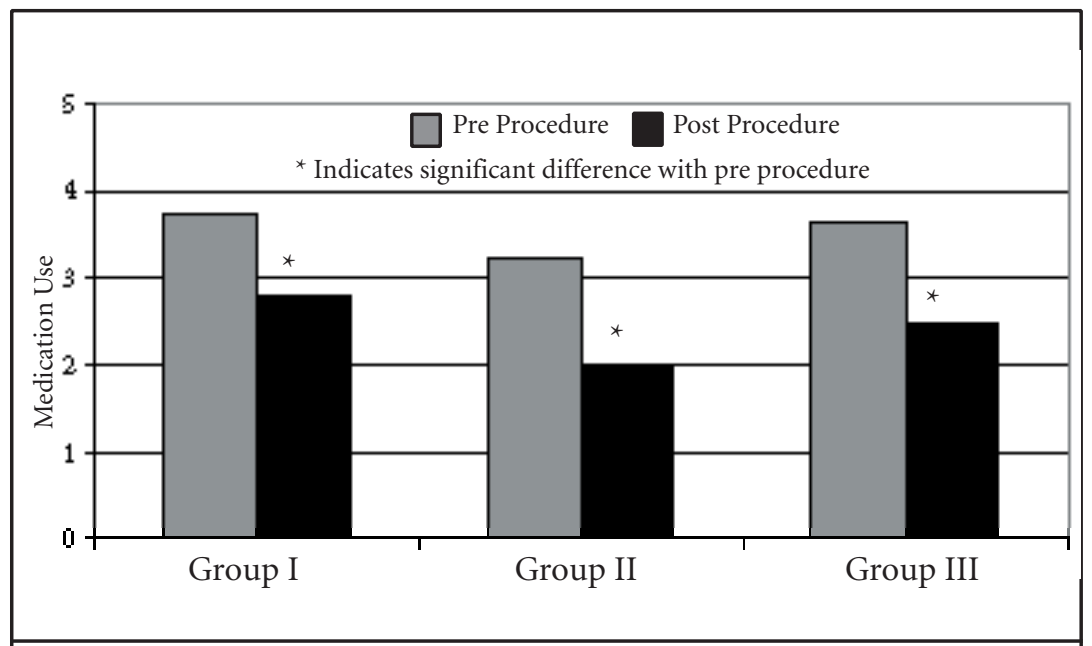

Fig. 4. Pain medication use double-blind study, due to ethical concerns and other logistical problems, randomized interventional studies are not widely performed. If we could repeat the study again, we would change the design to a prospective study where the data recording and analysis portions would be blinded to the researchers. By changing the design to such a study, we could eliminate one of the biggest weaknesses of our study - recall bias. We did not have the foresight to record patient baseline data during the pre-procedural office evaluation. While we agree that the study design would have been much stronger had we reduced potential recall bias, our mean baseline VAS pain score of 8.08 is consistent with other pain scores in at least one other study (8).

Our results may not show as great a reduction on the VAS pain scale because our inclusion criteria were less stringent. Several of the studies excluded patients with herniations greater than one-third the diameter of the spinal canal, while we excluded patients if they had herniations greater than one-half of the spinal canal diameter (8-10). While we still demonstrated a significant reduction in pain on the VAS scale, we may have shown an even greater reduction in pain and an even higher percentage of patients with at least a $50 \%$ reduction of pain had our criteria been less inclusive. By accepting patients with larger herniations, we included patients with more severe back problems who might not have found as much relief through nucleoplasty. The fact that we were able to demonstrate pain relief in this more complicated patient group illustrates that the nucleoplasty procedure shows promise as a therapeutic interventional technique.

\section{ConcLusion}

Our study shows statistically significant reductions in pain and the use of pain medications, and improvements in function as a result of using nucleoplasty to percutaneously decompress the disc. Nucleoplasty should be used in those patients who fail conservative medical management including medication, physical therapy, behavioral management, psychotherapy, and/or chiropractic treatment, and who are unwilling to undergo a more invasive technique such as spinal surgery. 


\section{AUthor AfFILATION:}

Arra S. Reddy, MD

Chief, Interventional Neuroradiology

Department of Radiology

Beth Israel Deaconess Medical Center

Harvard Medical School

One Deaconess Rd., Suite 580

Boston, MA 02215

E-mail: sreddy1@bidmc.harvard.edu

\section{Shaun Loh, BS}

Research Fellow

Department of Radiology

Beth Israel Deaconess Medical Center

Harvard Medical School

One Deaconess Rd., Suite 580

Boston, MA 02215

E-mail: sloh1@bidmc.harvard.edu

\section{Jennifer Cutts, MD}

Co-chief Resident, Residency Program Department of Radiology

Beth Israel Deaconess Medical Center

Harvard Medical School

One Deaconess Rd., Suite 580

Boston, MA 02215

E-mail: jcutts@bidmc.harvard.edu

\section{Jacob Rachlin, MD, PhD}

Staff Physician

Department of Neurosurgery

Beth Israel Deaconess Medical Center

Harvard Medical School

One Deaconess Rd., Suite 580

Boston, MA 02215

E-mail: jrachlin@caregroup.harvard.edu
Joshua A. Hirsch, MD

Radiology section editor, Pain

Physician journal

Director, Interventional

Neuroradiology / Endovascular

Neurosurgery

Chief, Minimally Invasive Spine

Surgery

Department of Radiology

Massachusetts General Hospital /

Harvard Medical School

55 Fruit St., GRB 289

Boston, MA 02114

E-mail: jahirsch@partners.org

\section{REFERENCES}

1. Brown D. Update on chemonucleolysis. Spine 1996; 21:62S-68S.

2. Nordby EJ, Wright PH, Schofield SR. Safety of chemonucleolysis. Adverse effects reported in the United States, 1982-1991. Clin Orthop Relat Res 1993; 293:122-134.

3. Ramberg N, Sahlstrand T. Early course and long-term follow-up after automated percutaneous lumbar discectomy. I Spinal Disord 2001; 14:511-516.

4. Onik G. Percutaneous lumbar diskectomy using a new aspiration probe. Am J Neuroradiol 1985; 6:290-296.

5. Choy DSJ, Ascher PW, Saddekni S, Alkaitus D, Liebler W, Hughes J, Diwan S, Altman P. Percutaneous laser disc decompression: A new therapeutic modality. Spine 1992; 17: 949-956.
6. Choy DSJ. Percutaneous laser disc decompression (PLDD): Twelve years experience with 752 procedures in 518 patients. J Clin Laser Med Surg 1998; 16:325-331.

7. Sherk HH, Black JD, Prodoehl JA, Cummings RS. Laser diskectomy. Orthopedics 1993; 16:573-576.

8. Sharps LS, Isaac Z. Percutaneous disc decompression using nucleoplasty ${ }^{\circledR}$. Pain Physician 2002; 5:121-126.

9. Singh V, Piryani C, Liao K, Nieschulz S. Percutaneous disc decompression using coblation (nucleoplasty ${ }^{\mathrm{TM}}$ ) in the treatment of chronic discogenic pain. Pain Physician 2002; 5:250-259.

10. Singh V, Piryani C, Liao K. Evaluation of percutaneous disc decompression using coblation in chronic back pain with or without leg pain. Pain Physician 2003; 6:
273-280.

11. Carragee EJ, Han MY, Suen PW, Kim D. Clinical outcomes after lumbar discectomy for sciatica: The effects of fragment type and annular competence. J Bone Joint Surg Am 2003; 85A:102-108.

12. Burton CV, Kirkaldy-Willis WH, Yong-Hing K, Heithoff KB. Cause of failure of surgery on the lumbar spine. Clin Orthop Relat Res 1981; 157:191-199.

13. Singh V, Piryani C, Liao K. Role of percutaneous disc decompression using coblation in managing chronic discogenic low back pain: A prospective, observational study. Pain Physician 2004; 7 : 419-425. 